\title{
Expression of DDR1 and DVL1 in Invasive Ductal and Lobular Breast Carcinoma does not Correlate with Histological Type, Grade and Hormone Receptor Status
}

\author{
Fereshteh Ameli, Isa Mohd Rose, Noraidah Masir*
}

\begin{abstract}
Background: Invasive ductal (IDC) and lobular (ILC) carcinomas are the common histological types of breast carcinoma which are difficult to distinguish when poorly differentiated. Discoidin domain receptor (DDR1) and Drosophila dishevelled protein (DVL1) were recently suggested to differentiate IDC from ILC. Objectives: To assess the expression of DDR1 and DVL1 and their association with histological type, grading and hormonal status of IDC and ILC. Materials and Methods: This cross sectional study was conducted on IDC and ILC breast tumours. Tumours were immunohistochemically stained for (DDR1) and (DVL1) as well as estrogen receptor $(E R)$, progesterone receptor $(\mathrm{PR})$ and $\mathrm{C}$-erbB2 receptor. Demographic data including age and ethnicity were obtained from patient records. Results: A total of 51 cases (30 IDCs and 21 ILCs) were assessed. DDR1 and DVL1 expression was not significantly associated with histological type $(p=0.57$ and $p=0.66$ respectively). There was no association between DDR1 and DVL1 expression and tumour grade ( $p=0.32$ and $p=1.00$ respectively), $E R$ ( $p=0.62$ and 0.50 respectively), $P R(p=0.38$ and $p=0.63$ respectively) and $C$-erbB2 expression $(p=0.19$ and $p=0.33$ respectively) in IDC. There was no association between DDR1 and DVL1 expression and tumour grade $(p=0.52$ and $p=0.33$ respectively), $E R(p=0.06$ and $p=0.76$ respectively), $P R(p=0.61$ and $p=0.43$ respectively) and C-erbB2 expression ( $p=0.58$ and $p=0.76$ respectively) in ILC. Conclusions: This study revealed that DDR1 and DVL1 are present in both IDC and ILC regardless of the tumour differentiation. More studies are needed to assess the potential of these two proteins in distinguishing IDC from ILC in breast tumours.
\end{abstract}

Keywords: Invasive breast cancer - DVL1 - DDR1 - expression

Asian Pac J Cancer Prev, 16 (6), 2385-2390

\section{Introduction}

Breast cancer is amongst the most prevalent cancers in the world accounting for $23 \%$ of new cases of cancer and is responsible for $14 \%$ of mortality due to cancer (Jemal et al., 2011; Abdullah et al., 2013). The annual incidence of breast cancer is reported to increase by $2 \%$ worldwide regardless of the income level of the countries (Bray et al., 2012; Alsanabani et al., 2015; Varughese et al., 2015). It is estimated that the risk of developing invasive breast cancer in life time is $12.3 \%$ for American women (DeSantis et al., 2014). In Malaysia, breast cancer is the most common cancer which accounts for $32.1 \%$ of total female cancers (Omar andTamin, 2011). Moreover, it was reported that $24.2 \%$ of diagnosed breast cancers in Malaysia were in stage III and $17.7 \%$ in stage IV (Omar and Tamin, 2011).

The most common histological types of breast carcinoma include invasive ductal carcinoma (IDC) and invasive lobular carcinoma (ILC) upon which IDC comprises about $80 \%$ of the breast carcinomas (Keller et al., 2012; Hanley, 2014). A previous study in Malaysia and
Singapore the most common histological type of breast cancer was ductal $(87 \%)$ followed by other types $(9 \%)$ and lobular (4\%) (Bhoo Pathy et al., 2011).

Both IDC and ILC may have similar presentation in terms of tumor site, size, grade and stage; however, clinical follow-up data and the patterns of metastasis suggest that the development and progression of these two types of breast carcinoma are different (Turashvili et al., 2007).

ILC is defined as a low-grade tumor with little or no nuclear atypia and low mitotic rate. Unlike IDCs, ILC has ill-defined margins and does not form microcalcifications, making it difficult to detect on screening mammography and ultrasound (Yoder et al., 2007). In ILC tumor cells tend to infiltrate beyond the palpable extent of the tumor, therefore, resection margins are more frequently tumor positive and recurrence rates are higher than IDC (Lehret al., 2000). ILCs have unique patterns of metastasis compared to IDCs. ILCs have a higher tendency to occur bilateral and to metastasize to the peritoneum, gastrointestinal system, gynecologic organs, bone marrow, and leptomeninges than IDCs but the extension to lung is 
Fereshteh Ameli et al

more frequent in IDCs compared to ILCs (Yehet al., 2011; Dey et al., 2013). Overall, ILC has a better prognosis compared with ductal carcinoma not otherwise specified (NOS) (Majeed et al., 2014). Moreover, the incidence of ILC was shown to be steadily increasing over the past 20 years, while its precise cause remains unknown (Yoder et al., 2007). Existing data support the theory that ILC might be related to the use of hormone replacement therapy among postmenopausal women (Yoder et al., 2007).

Both IDC and ILC originate from terminal duct lobular unit (TDLU) (Figueroa et al., 2014). Although both tumours have a similar origin, their underlying genetic causes are different. Therefore, these tumours have different cellular and molecular characteristics that can be used for discriminating one from another (Yeh et al., 2011; Dey et al., 2013).

Typical ductal carcinomas are distinctly positive for E-cadherin, but lobular carcinomas usually do not express membrane positivity for E-cadherin (Moriya et al., 2009). Although E-cadherin membranous staining positivity usually indicates ductal differentiation of carcinoma, less than $15 \%$ of IDCs, mainly poorly differentiated carcinoma, lack E-cadherin immunoreactivity (Moriya et al., 2009). These E-cadherin negative IDCs were shown to have a worse prognosis compared with E-cadherin positive IDCs (Moriya et al., 2009). On the other hand, E-cadherin positivity may be present in $10 \%$ of ILCs, therefore, immunophenotyping remains ambiguous in minority of cases (Moriya et al., 2009).

Discoidin domain receptor 1 (DDR1) and dishevelled segment polarity protein 1 (DVL1) are recently described proteins that are hypothesized to be able to differentiate IDC from ILC (Turashvili et al., 2007; Kafka, et al., 2014). DDR 1 is a tyrosine receptor kinase activated by collagen and is involved in cell-matrix communication. The physiological functions of DDR 1 are not clearly understood but DDR1 is shown to have a role in cell interactions with the extracellular matrix by controlling adhesion and cell motility (Quanet al., 2011). DVL1 is an essential mediator of both canonical and noncanonical Wnt pathways. The Wnt proteins belong to a large family of secreted signaling molecules that are implicated at several stages of mammary gland growth and differentiation (Turashvili et al., 2007). Amplification and increased expression of the DVL1 gene have been observed in $50 \%$ of the breast carcinomas and therefore were found to be associated with breast carcinogenesis (Band et al., 2011; Dey et al., 2013a; Dey et al., 2013b). To the best of our knowledge no study has been conducted on the assessment of DDR1 and DVL1 expression in Malaysian population. The aim of this study was to assess the expression of DDR1 and DVL1 in breast carcinomas and their association with histological and hormonal status of the breast carcinomas.

\section{Materials and Methods}

This cross sectional study was conducted on the newly diagnosed IDC and ILC obtained from histopathology unit of the pathology Department, University Kebangsaan Malaysia Medical Centre from $1^{\text {st }}$ January 2002 to $31^{\text {st }}$ December 2010. Samples were selected from the specimens that were previously evaluated for estrogen receptor (ER), Progesterone receptor (PR) and C-erbB-2 status. Patients who started or were on chemotherapy or radiotherapy were excluded from the study.

All histological slides and paraffin embedded blocks were retrieved. All the cases were reviewed independently by two observers, the pathologist and trainee pathologist. The clinicopathological variables obtained from the histopathological data included tumour grading, ER, PR, and C-erbB-2 status. Samples were reassessed in field diameter of $0.65 \mathrm{~mm}$, at the high field diameter of $400 \mathrm{X}$ (Olympus BX40 and BX41, Japan).

Tumour grading was based on the Nottingham modification of the Bloom-Richardson system, which also incorporates the evaluation of mitotic activity in addition to architectural features (extent of tubular formation) and the degree of nuclear atypia by Bloom and Richardson and Black (Feldman and Eunhee, 2012). In this scheme, the grade is obtained by the summation of scores for tubule formation, nuclear pleomorphism and mitotic count (scores range from 1 to 3 resulting in a total score between 3 and 9). Scores between 3 and 5 are considered as grade 1 , scores between 6 and 7 as Grade II and scores between 8 and 9 as Grade III. Ten cases of each grade were collected for both IDC and ILC. There was no ILC grade 3 identified due to biology of this tumour, which is usually a low-grade tumour.

Determination of ER, PR and C-erbB2 staining were done by the reporting pathologists and the results were obtained from the histopathological records. Staining of the ER and PR were evaluated in the nuclei of the malignant cells and were scored as positive or negative. Tumours were considered to be positive for ER and PR when more than $1 \%$ of the neoplastic cells were stained at any intensity. If less than $1 \%$ of the nuclei were stained, the tumour was considered as negative (Ferrero-Poüs et al., 2001).

The overexpression of C-erbB2 was measured by immunohistochemical study. The interpretation was performed based on the recommendation by the American Society of Clinical Oncology (ASCO) and the College of American Pathologists (CAP) guidelines(Feldman and Eunhee, 2012). For determination of C-erbB-2 overexpression, only the membranous staining was observed and the intensity of the tumour cells was scored (Feldman and Eunhee, 2012).

\section{Antibody and immunohistochemistry}

Rabbit Polyclonal anti-human MCK10 or DDR1 (DAKO, Dilution 1:200)and anti-human to DVL1 (DAKO, Dilution 1:50)were used for immunohistochemical staining. Both antibodies were obtained from Abcam Company, USA (Code No. ab5508 and ab21062).

\section{Immunohistochemistry}

Immunohistochemical staining was performed on the paraffin tissue section slides. The staining conditions were adjusted according to previous data from literature (Turashvili et al., 2007). Staining was performed using the protocols from Dako REAL ${ }^{\mathrm{TM}}$ EnVision $^{\mathrm{TM}}$ Detection 
System, Peroxidase/DAB+, Rabbit/Mouse (Code No. K5007). Primary antibodies were diluted using Antibody Diluent (DAKO) to optimal concentration. Washing steps between each reagents were performed using Tris Buffered Saline (TBS) \& Tween 20 (20x) from Lab Vision Corporation diluted to a $1 \mathrm{x}$ working solution with distilled water. The 1x DAB-containing Substrate Working Solution was prepared by diluting the $50 \mathrm{x}$ concentrated Dako REAL ${ }^{\mathrm{TM}}$ DAB+ chromogen with DakoREAL ${ }^{\mathrm{TM}}$ Substate Buffer.

Tissue slides were incubated at $65^{\circ} \mathrm{C}$ on hot plate for 30 minutes. An initial dewaxing step was performed using 2 times xylene and rehydration step using decreasing alcohol solutions $(100 \%, 80 \%$ and $70 \%)$. Then the slides were rinsed in running tap water for 3 minutes. The slides were subsequently incubated with hydrogen peroxide $\left(\mathrm{H}_{2} \mathrm{O}_{2}\right)$ for 5 minutes and were then rinsed with running tap water. Antigen retrieval step was performed using heat-induced antigen retrieval $\mathrm{pH} 9.0$ Tris-ethylenediaminetetraceticacid (EDTA)-based solution (DAKO)in the Pascal Pressurized Chamber (DakoCytomation, USA) at $97^{\circ} \mathrm{C}$ for 40 minutes followed by cooling at room temperature for 20 minutes and rinsing with running tap water. Slides were then incubated for overnight at room temperature with primary antibody MCK10 (dilution 1:200) and DVL1 (dilution 1:50). Slides were then incubated with Dako REAL ${ }^{\mathrm{TM}}$ EnVision $^{\mathrm{TM}} / \mathrm{HRP}$, Rabbit/Mouse (ENV) for 30 minutes. Sections were then incubated with $1 \mathrm{x}$ DAB-containing Substrate Working Solution for 5 minutes. Hematoxylin counterstaining (30 seconds) was performed after the procedures have been completed followed by dehydration, clear and mounting steps using DPX mounting medium.

\section{Controls}

Positive controls from normal salivary gland and gastric tissue were used as controls in order to confirm the consistency of the analyses. For the negative control, the same sections were incubated omitting the primary antibody.

\section{Interpretation of the results}

After staining, the slides were reviewed by two observers (pathologist and pathologist trainee). Representative fields were selected and topographic localization (membrane and/or cytoplasmic) of DDR1 and DVL1 expression were evaluated. The percent of all positively stained tumor cells out of 1000 tumor cells were recorded (Turashvili et al., 2007).The determinations were made on high magnification (X400) in the areas with the greatest degree of immunostaining.

\section{Statistical analysis}

Data collected was entered into a computer file and statistically analyzed using statistical package for social sciences (SPSS) software version 19.0 (IBM Inc, Chicago, Il, USA). The result was analyzed statistically by using Fisher's Exact test or Chi Square.The statistical significance was set as $\mathrm{p}<0.05$.

This study was approved by the Ethical and Research Committee, Faculty of Medicine, UniverstiKebangsaan Malaysia Medical Centre (UKMMC).

\section{Results}

A total of 51 cases (30 IDCs and 21 ILCs) were included in this study. The average age of the cases was $55 \pm 12.5$ years (ranging from 34 to 84 years). Mean age was younger for ILC 52 years compare with IDC 58 years. All the patients were female. Demographic characteristics of the cases are shown in Table 1. There was no significant difference between IDC and ILC cases in terms of demographic characteristics (Table 1).

\section{Association between DDR1 and DVL1 expression and Histological type of tumours}

The correlation of DDR 1 and DVL1 expression with histological type of tumour is shown in (Table1). There was no significant association between DDR1 and DVL1 positivity and histological type of breast carcinoma (Table 1 and Figure 1).

Association between DDR1 and DVL1 expression and Histological grade of tumours

The relationship of DDR 1 and DVL1 expression with histological grade is shown in (Table 2 and 3 ). There was no significant association between DDR1 and DVL1 positivity and histological type of breast carcinoma (Table 2 and 3$)$.

Table 1. Demographics of Patient and Tumour Characteristics for IDC

\begin{tabular}{|c|c|c|c|}
\hline \multirow[t]{2}{*}{ Variables } & \multicolumn{2}{|c|}{ Tumor type $(\mathrm{N}=51)$} & \multirow[t]{2}{*}{$\mathrm{p}$} \\
\hline & $\begin{array}{c}\text { IDC } \\
(\mathrm{n}=30)(\%)\end{array}$ & $\begin{array}{c}\text { ILC } \\
(\mathrm{n}=21)(\%)\end{array}$ & \\
\hline \multicolumn{4}{|l|}{$\overline{\text { Age (years) }}$} \\
\hline $30-40$ & $7(23.3)$ & - & 0.08 \\
\hline $40-50$ & $7(23.3)$ & $5(23.8)$ & \\
\hline $50-60$ & $8(26.7)$ & $10(47.6)$ & \\
\hline$>60$ & $8(26.7)$ & $6(28.6)$ & \\
\hline \multicolumn{4}{|l|}{ Ethnicity } \\
\hline Malay & $20(66.7)$ & $11(52.4)$ & 0.58 \\
\hline Chinese & $8(26.7)$ & $9(42.9)$ & \\
\hline Indian & $2(6.7)$ & $1(4.8)$ & \\
\hline \multicolumn{4}{|l|}{ ER } \\
\hline Negative & $15(50)$ & $5(23.8)$ & 0.08 \\
\hline Positive & $15(50)$ & $16(76.2)$ & \\
\hline \multicolumn{4}{|l|}{ PR } \\
\hline Negative & $19(63.3)$ & $9(42.9)$ & 0.17 \\
\hline Positive & $11(36.7)$ & $12(57.1)$ & \\
\hline \multicolumn{4}{|l|}{ C-erbB-2 } \\
\hline Negative & $20(66.7)$ & $16(76.2)$ & 0.54 \\
\hline Positive & $10(33.3)$ & $5(23.8)$ & \\
\hline \multicolumn{4}{|l|}{ DDR1 } \\
\hline Negative $($ Score $0 \leq 10 \%)$ & $5(16.7)$ & $3(14.3)$ & 0.57 \\
\hline Positive: & $25(83.3)$ & $18(85.7)$ & \\
\hline Score1 $(11-25 \%)$ & 1(3.3) & $1(4.8)$ & \\
\hline Score2 $(26-75 \%)$ & $6(20)$ & $1(4.8)$ & \\
\hline Score $3(>75 \%)$ & $18(60)$ & $16(76.2)$ & \\
\hline \multicolumn{4}{|l|}{ DVL1 } \\
\hline Negative $($ Score $0 \leq 10 \%)$ & $1(3.3)$ & $1(4.7)$ & 0.66 \\
\hline Positive: & $29(96.6)$ & $20(95.3)$ & \\
\hline Score1 (11-25\%) & $1(3.3)$ & 1(4.8) & \\
\hline Score2 $(26-75 \%)$ & $1(3.3)$ & $0(0)$ & \\
\hline Score $3(>75 \%)$ & $27(90.3)$ & $19(90.5)$ & \\
\hline
\end{tabular}

*Fisher exact test was used 


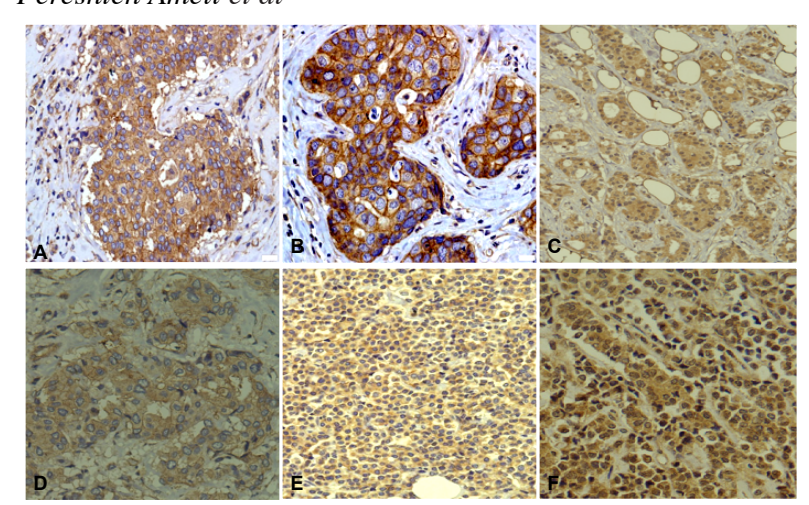

Figure 1. Association between DDR1 and DVL1 expression and Histological type and grade of tumours Cytoplasmic and Membrane Staining of DDR1 in IDC Grade 1 (X400) (A). Cytoplasmic and membrane staining of DDR1 in IDC grade 3 (X400) (B). Cytoplasmic staining of DVL1 in IDC grade 1 (X200) (C).Cytoplasmic staining of DVL1 in IDC grade 3 (X400) (D). Cytoplasmic staining of DDR1 in ILC grade 2 (X400) (E). Cytoplasmic staining for DVL1 in ILC grade 2 (X400) (F).

Table 2. Association between DDR1 and DVL1 Expression and Grade of Tumours, ER, PR and C-erbB2 Expression in IDC Samples $(\mathbf{n}=30)$

\begin{tabular}{|c|c|c|c|c|c|c|}
\hline & \multicolumn{3}{|c|}{ DDR1 } & \multicolumn{3}{|c|}{ DVL1 } \\
\hline & $\overline{\text { Preserved }}$ & Absent & $\overline{t p}$ & Preserved & Absent & $\overline{\mathrm{p}}$ \\
\hline \multicolumn{7}{|l|}{ Grade } \\
\hline I & 10 & 0 & 0.32 & 10 & 0 & 1 \\
\hline II & 8 & 2 & & 9 & 1 & \\
\hline III & 7 & 3 & & 10 & 0 & \\
\hline \multicolumn{7}{|l|}{ ER } \\
\hline Positive & 13 & 2 & 0.62 & 14 & 1 & 0.5 \\
\hline Negative & 12 & 3 & & 15 & 0 & \\
\hline \multicolumn{7}{|l|}{ PR } \\
\hline Positive & 10 & 1 & 0.38 & 11 & 0 & 0.63 \\
\hline Negative & 15 & 4 & & 18 & 1 & \\
\hline \multicolumn{7}{|l|}{ CerbB2 } \\
\hline Positive & 7 & 3 & 0.19 & 9 & 1 & 0.33 \\
\hline Negative & 18 & 2 & & 20 & 0 & \\
\hline
\end{tabular}

*Fisher exact test was used

Table 3. Association between DDR1 and DVL1 Expressions and Grade of Tumours, ER, PR and C-erbB2 Expression in ILC Samples $(\mathbf{n}=21)$

\begin{tabular}{|c|c|c|c|c|c|c|}
\hline & \multicolumn{3}{|c|}{ DDR 1} & \multicolumn{3}{|c|}{ DVL1 } \\
\hline & $\overline{\text { Preserved }}$ & Absent & $\bar{p}$ & Preserved & Absent & $\mathrm{p}$ \\
\hline \multicolumn{7}{|l|}{$\overline{\text { Grade }}$} \\
\hline I & 11 & 3 & 0.52 & 14 & 0 & 0.33 \\
\hline II & 7 & 0 & & 6 & 1 & \\
\hline III & 0 & 0 & & 0 & 0 & \\
\hline \multicolumn{7}{|l|}{ ER } \\
\hline Positive & 15 & 1 & 0.06 & 15 & 1 & 0.76 \\
\hline Negative & 3 & 2 & & 5 & 0 & \\
\hline \multicolumn{7}{|l|}{ PR } \\
\hline Positive & 10 & 2 & 0.61 & 12 & 0 & 0.43 \\
\hline Negative & 8 & 1 & & 0 & 1 & \\
\hline \multicolumn{7}{|l|}{ C-erbB2 } \\
\hline Positive & 4 & 1 & 0.58 & 5 & 0 & 0.76 \\
\hline Negative & 14 & 2 & & 15 & 1 & \\
\hline
\end{tabular}

*Fisher exact test was used
Association between DDR1 and DVL1 expression and $E R, P R, C$-erbB2

The correlation of DDR 1 and DVL1 expression with hormonal status is shown in (Table 2 and 3). There was no significant association between DDR1 and DVL1 positivity and hormonal status in breast carcinoma (Table 2 and 3$)$.

\section{Discussion}

This study showed that the incidence of breast cancer is high in middle-aged population. This peak incidence of breast cancer does not differ significantly from that reported in cancer incidence in Peninsular Malaysia in which the peak incidence occurred in the 50-60 years age group (Omar and Tamin, 2011).

The ethnic distribution of breast cancer in our study showed that Malay women appear to be more commonly affected, followed by Chinese and Indians. These data were in contrast from the previously incidence rates reported by the cancer incidence in Peninsular Malaysia, where breast cancer was most common in Indian women (36\%) followed by Malay (33.8\%) and Chinese (33.7\%) (Omar andTamin, 2011). This difference might be due to the small sample size of this study since this study was not intended to identify the incidence rate of breast carcinoma in Malaysian population.

ER and PR positivity was found to be higher in ILC compared with IDC but c-erbB-2 overexpression in ILC was lower than IDC which is in line with previous studies which showed that ILC tumours are more ER and PR positive and C-erbB2 negative (Russo et al., 2001; Hussainand Cunnick, 2011; Man et al., 2011; Tiedeand Kang, 2011).

In this study no significant association was found between DDR1 and DVL1 expression and histological type of breast cancer. There is controversy in the findings of previous studies regarding DDR1 and DVL1 expression in IDC and ILC (Turashvili et al., 2007). Turashvili et al. (2007) reported DDR 1 expression in $96.2 \%$ of IDC cases and $13.8 \%$ of ILC cases and DVL1 expression in $25 \%$ of IDC and $96.5 \%$ of ILC tumours which was different from the findings of this study. This discrepancy may be explained in part by the difference in sample size of the studies, different antibody agents and different techniques that were applied. The animal source of antibodies (rabbit polyclonal) in this study was different from Turashvili's study (mouse polyclonal)(Turashvili et al., 2007). In general, rabbit polyclonal antibodies are more efficient and advantageous than those of mice or other rodents because the rabbit immune system generates antibody diversity and optimizes affinity by mechanisms that are more efficient than those of mice and other rodents (Rocha et al., 2008). In addition, the antibody dilution that was optimized in this study (DDR1 1:200 and DVL1 1:50 with overnight incubation at room temperature) also differed from Turashvili's study (1:100 for both DDR1 and DVL1) (Turashvili et al., 2007). Moreover, the exact role and expression of these antibodies in breast cancer is still being investigated. However biological difference between Malaysian and Jewish population could be 
another reason for this discrepancy. In contrast, a study conducted on Chinese population by Yin et al. (2009) reported that DVL1 positivity rate was $54.2 \%(13 / 24)$ in IDC group and $41.6 \%$ (5/12) for ILC. They highlighted the limited value of DVL1 for differentiation between ILC and IDC which was in agreement with the findings of this study (Yin et al., 2009).

This present study was the first study to compare the association between DDR1 and DVL1 expression and tumour differentiation grade, ER, PR and C-erbB2 in breast carcinomas. This study revealed that the expression of DDR1 and DVL1 was independent of tumour differentiation grade, ER, PR and C-erbB 2 status in both IDC and ILC tumours.

Further studies based on molecular or cytogenetic on larger samples are desirable to establish the basic role of these proteins in normal and malignant breast tissues. With regards to distinction between IDC and ILC, further investigations are needed to find a more reliable and novel diagnostic marker or panel of markers that could support both the pathologists and clinicians in improving therapeutic decisions and management for these two types of breast cancer.

This study proved that DDR1 and DVL1 are not reliable markers for differentiation between IDC and ILC. E-cadherin is still the only well-established immunohistochemical marker for this differentiation.

\section{Acknowledgements}

This study was funded by the Faculty of Medicine, UniversitiKebangsaan Malaysia and Science Fund (project code: FF-104-2010).

\section{References}

Abdullah NA, Wan Mahiyuddin WR, Muhammad NA, et al (2013). Survival rate of breast cancer patients in Malaysia: a population based study. Asian Pac J Cancer Prev, 14, 4591-4.

Alsanabani JA, Gallan W, Saadi AA (2015). Incidence data for breast cancer among Yemeni female patients with palpable breast lumps. Asian Pac J Cancer Prev, 16, 191-4.

Band V, Zhao X, Malhotra G, et al (2011).Shared signaling pathways in normal and breast cancer stem cells. $J$ Carcinogenesis, $\mathbf{1 0}, 38$.

BhooPathy N, Yip CH, Taib NA, et al (2011). Breast cancer in a multi-ethnic Asian setting: Results from the SingaporeMalaysia hospital-based breast cancer registry. Breast, 20, 75-80.

Bray F, JemalA, Grey N, et al (2012). Global cancer transitions according to the human development index (2008-2030): a population-based study. Lancet Oncology, 13, 790-801.

DeSantis C, Ma J, Bryan L, et al (2014). Breast cancer statistics, 2013. CA-Cancer J Clin, 64, 52-62.

Dey N, Barwick BG, Moreno CS, et al (2013a).Wnt signaling in triple negative breast cancer is associated with metastasis. BMC Cancer, 13, 537.

Dey N, Young B, Abramovitz M, et al (2013b). Differential activation of Wnt- $\beta$-Catenin pathway in triple negative breast cancer increases MMP7 in a PTEN dependent manner. PLoS ONE, 8, 77425.

FeldmanAL,Eunhee SY (2012). Rosai and ackerman's surgical pathology. JAMA, 307, 201-2.

Ferrero-Pous M, Trassard M, Le Doussal V, et al (2001). Comparison of enzyme immunoassay and immunohistochemical measurements of estrogen and progesterone receptors in breast cancer patients.Appl Immunohisto Mol Morph, 9, 267-75.

Figueroa JD, Pfeiffer RM, Patel DA, et al (2014). Terminal duct lobular unit involution of the normal breast: implications for breast cancer etiology. J Natl Cancer Inst, 106, 286.

Hanley KZ (2014). Rosen's Breast Pathology. LWW.

HussainM,Cunnick GH (2011). Management of lobular carcinoma in-situ and atypical lobular hyperplasia of the breast--a review. Eur J Surg Oncol, 37, 279-89.

Jemal A, Bray F, Center MM, et al (2011). Global cancer statistics. CA-Cancer J Clin, 61, 69-90.

Kafka A, Basic-KindaS, Pecina-Slaus N (2014). The cellular story of dishevelleds. Croatian Med J, 55, 459-67.

Keller PJ, Arendt LM, Skibinski A, et al (2012). Defining the cellular precursors to human breast cancer. Proc Natl Acad Sci USA, 109, 2772-7.

Lehr HA, Folpe A, Yaziji H, et al (2000). Cytokeratin 8 immunostaining pattern and E-cadherinexpression distinguish lobular from ductal breast carcinoma. Am J Clin Pathol, 114, 190-6.

Majeed W, Aslam B, Javed I, et al (2014). Breast cancer: major risk factors and recent developments in treatment. Asian Pac J Cancer Prev, 15, 3353-8.

Man Y, Izadjoo M, Song G, et al (2011). In situ malignant transformation and progenitor-mediated cell budding: two different pathways for breast ductal and lobular tumor invasion. Cancer, 2, 401-12.

Moriya T, Kozuka Y, Kanomata N, et al (2009). The role of immunohistochemistry in the differential diagnosis of breast lesions. Pathology, 41, 68-76.

OmarZA,Tamin NSI (2011). National cancer registry report: malaysia cancer statistics-data and figure 2007. national cancer registry, Ministry of Health Malaysia.

Quan J, Yahata T, Adachi S, et al (2011). Identification of receptor tyrosine kinase, discoidin domain receptor 1 (DDR1), as a potential biomarker for serous ovarian cancer. Intern $\mathrm{J} \mathrm{Mol}$ Sci, 12, 971-82.

Rocha R, Nunes C, Rocha G, et al (2008). Rabbit monoclonal antibodies show higher sensitivity than mouse monoclonals for estrogen and progesterone receptor evaluation in breast cancer by immunohistochemistry. Pathol Res Pract, 204, 655-62.

Russo J, Lynch H, Russo IH (2001). Mammary gland architecture as a determining factor in the susceptibility of the human breast to cancer. Breast, 7, 278-91.

Tiede B, Kang Y (2011). From milk to malignancy: the role of mammary stem cells in development, pregnancy and breast cancer. Cell Res, 21, 245-57.

Turashvili G, Bouchal J, Baumforth K, et al (2007). Novel markers for differentiation of lobular and ductal invasive breast carcinomas by laser microdissection and microarray analysis. BMC Cancer, 7, 55.

Vaurghese AA, Poothiode U, Manjula V (2015). Descriptive study on selected risk factors and histopathology of breast carcinoma in tertiary care centre in Kerala, India with special reference to women uder 40 years old. Asian Pac J Cancer Prev, 16, 181-4.

Yeh YC, Wu CC, Wang YK, et al (2011). DDR1 triggers epithelial cell differentiation by promoting cell adhesion through stabilization of E-cadherin. MolBiol Cell, 22, 940-53.

Yin HF, Li T, Zhang H, et al (2009). Differential diagnosis of invasive ductal carcinoma versus invasive lobular carcinoma 
Fereshteh Ameli et al

of breast. Chinese J Pathol, 38, 663-7.

Yoder BJ, Wilkinson EJ, Massoll NA (2007). Molecular and morphologic distinctions between infiltrating ductal and lobular carcinoma of the breast. Breast, 13, 172-9. 